\title{
Recomposition des collectifs agricoles pour des filières plus durables
}

Reshaping agricultural collectives for more sustainable value chains

Hichem Amichi, Julien Frayssignes, Marie-Christine Henninger et Arnaud Buchs

\section{(2) OpenEdition}

1 Journals

\section{Édition électronique}

URL : https://journals.openedition.org/developpementdurable/19064

DOI : 10.4000/developpementdurable.19064

ISSN : 1772-9971

Éditeur

Association DD\&T

\section{Référence électronique}

Hichem Amichi, Julien Frayssignes, Marie-Christine Henninger et Arnaud Buchs, « Recomposition des collectifs agricoles pour des filières plus durables », Développement durable et territoires [En ligne], Vol. 12, $n^{\circ} 1$ | Mai 2021, mis en ligne le 26 mai 2021, consulté le 28 mai 2021. URL : http:// journals.openedition.org/developpementdurable/19064; DOI : https://doi.org/10.4000/ developpementdurable.19064

Ce document a été généré automatiquement le 28 mai 2021.

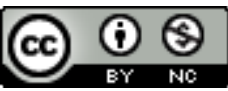

Développement Durable et Territoires est mis à disposition selon les termes de la licence Creative Commons Attribution - Pas d'Utilisation Commerciale 4.0 International. 


\title{
Recomposition des collectifs agricoles pour des filières plus durables
}

\author{
Reshaping agricultural collectives for more sustainable value chains
}

Hichem Amichi, Julien Frayssignes, Marie-Christine Henninger et Arnaud Buchs

Les auteurs tiennent à remercier les évaluateurs et évaluatrices anonymes pour leurs relectures constructives qui ont permis d'améliorer les articles de ce dossier thématique. Les auteurs remercient également l'École d'ingénieurs de PURPAN pour son soutien tant sur le plan organisationnel que financier.

1 Dans un contexte économique changeant et fortement concurrentiel, l'agriculture française fait face à l'émergence de nouveaux enjeux. Ces enjeux sont liés aux préoccupations environnementales, aux questions de viabilité économique d'exploitations agricoles et à leur maintien au sein des territoires ruraux, à l'apparition de nouvelles attentes, relatives par exemple à la qualité des produits agricoles, au "consommer local», à la rémunération des producteurs et aux modes de commercialisation. Dans ce contexte, l'hétérogénéité de l'agriculture est croissante au sein des territoires, influencée par une tendance forte à la diversification des stratégies de production, de transformation, de commercialisation et de leurs fonctions associées (Hervieu, 2002 ; Van Der Ploeg et al., 2009).

2 Pour répondre à ces transformations, le recours aux collectifs constitue un des leviers privilégiés car ils permettent d'inscrire les acteurs - les agriculteurs en premier lieu au centre des processus de transition agricole (Demeulenaere et Goulet, 2012 ; Lucas et al., 2014). La consécration du groupe comme vecteur ou levier de la transformation agricole n'est pas nouvelle. Les premiers groupements d'échanges entre agriculteurs créés et formalisés, en lien avec la transformation agricole, remontent à la période de l'après-guerre, à l'image de la Jeunesse agricole catholique (JAC, qui a vu son rôle évoluer en cette période vers une mission d'accompagnement de la modernisation 
agricole [Flauraud, 2005]) et des Centres d'études techniques agricoles (Ceta). Ces collectifs ont inscrit durablement la notion de groupe au sein de la vulgarisation agricole française et ont permis de responsabiliser les agriculteurs en les amenant à penser par eux-mêmes la transformation de leur exploitation (Gerbeaux et Muller, 1984). Ainsi, le processus de spécialisation et d'intensification de la production agricole a été accompagné par la coopération agricole dans ses formes diverses, qu'elles soient de type coopératif, associatif ou sociétaire.

3 Dans le contexte actuel de transition, qualifié par certains auteurs de postproductivisme (Van Der Ploeg, 2014), ces structures "traditionnelles» de la coopération agricole présentent un certain nombre de limites et peinent à s'adapter aux nouvelles transformations du monde agricole. La principale raison réside dans le fait que ces structures restent, du moins aux yeux d'une partie des agriculteurs et d'autres acteurs locaux, fortement ancrées dans le modèle dit productiviste de l'agriculture. La défiance des agriculteurs vis-à-vis de ces structures se manifeste, par exemple, au sein des adhérents des coopératives qui déclarent ne plus retrouver les valeurs fondamentales de la coopération et perdent ainsi les liens historiques avec leurs structures (Barraud Didier et al., 2012). Certaines coopératives connaissent une forte distanciation des rapports avec leurs adhérents en raison d'une quête d'efficacité économique les conduisant à s'agrandir par des processus de fusion et de filialisation (Filippi et al., 2008). Si cette défiance vis-à-vis de la coopération se vérifie moins pour les Coopératives d'utilisation de matériel agricole (Cuma) par rapport aux autres groupes coopératifs, certains membres expriment une volonté de s'émanciper d'une organisation encore marquée par son héritage "modernisateur» (Lucas et al., 2014). Des démarches de diversification agricole, de transformation ou de commercialisation de type "circuits courts ", sont entreprises et inscrivent plutôt leurs porteurs sur la voie de formes alternatives d'agriculture (Allaire et Assens, 2012 ; Lucas et al., 2014).

Cette recomposition des collectifs en agriculture se manifeste soit par le repositionnement des anciennes structures qui essayent de repenser leurs modalités de fonctionnement et de redéfinir les liens avec leurs membres, soit par l'émergence de nouveaux groupes qui tentent d'explorer d'autres manières de faire du collectif. La diversité de ces initiatives collectives a été documentée par des travaux scientifiques récents. Ils témoignent d'une volonté d'évoluer vers des modèles de productions agricoles qui intègrent, en plus des préoccupations économiques (Richard et al., 2014 ; Bouroullec, 2020), les dimensions sociales (rapport au local, juste rémunération des producteurs, etc. [Chiffoleau et Paturel, 2016]) et environnementales (préservation des ressources naturelles, réductions d'intrants chimiques, etc. [Maréchal et Spanu, 2010]). Ces travaux portent essentiellement sur deux dimensions de l'activité agricole. Il s'agit d'une part des collectifs qui réfléchissent aux modalités d'adoption de nouvelles pratiques agricoles (les collectifs en agriculture bio par exemple [Van Dam et al., 2017]). Il s'agit d'autre part des collectifs qui explorent et expérimentent de nouvelles formes de commercialisation proches du consommateur (la dynamique des circuits courts collectifs notamment [Chiffoleau et Prevost, 2012]). Cependant, à l'exception de quelques auteurs (Vincq et al., 2010 ; Mundler et Valorge, 2015 ; Gafsi et Delevers, 2015), très peu de recherches sont consacrées aux collectifs relatifs à d'autres dimensions de l'activité agricole, telles que la transformation, l'abattage, les activités de logistique.

5 Ce dossier thématique a donc deux ambitions principales. Il vise à recueillir des contributions originales qui rendent compte de la reconfiguration actuelle de ces 
collectifs touchant à différentes dimensions de l'activité agricole, en plus de la production et de la commercialisation. Il vise également à questionner le rôle de ces collectifs dans le maintien ou le développement d'exploitations et de filières agricoles durables. Pour ce faire, les contributions de ce dossier articulent travaux empiriques et approches pluri- (voire inter-) disciplinaires afin d'apporter des éclairages suivant quatre axes de réflexion.

6 Le premier axe de réflexion met en évidence la diversité des dynamiques d'émergence des collectifs. La création de ces collectifs ou leur renouvellement n'émanent pas uniquement de producteurs ou de consommateurs. Si les dimensions économiques restent bien évidemment centrales pour expliquer l'émergence des collectifs (recherche d'une meilleure valorisation économique des produits par exemple), de nouvelles attentes s'expriment vis-à-vis de ces derniers, telles que l'autonomie vis-à-vis des structures "traditionnelles» ou du régime sociotechnique dominant, et la recherche d'espaces de convivialité et de socialisation.

7 Le deuxième axe de réflexion questionne la gouvernance de ces collectifs et leur pérennité. Malgré une définition et une coconstruction de règles de fonctionnement claires au sein de ces groupes, des questions restent posées en matière de fonctionnement, en lien notamment avec les problématiques de fidélisation et d'implication des membres.

8 Le troisième axe traite du rapport des collectifs au territoire et interroge leurs stratégies à travers ce prisme. Il questionne notamment la manière dont les formes de rapports aux territoires - récentes ou plus anciennes - constituent pour ces collectifs des opportunités en termes de compétitivité et de pérennité.

Le quatrième axe documente la diversité des dispositifs institutionnels régionaux mis en place afin de s'adapter et d'accompagner la recomposition des collectifs au sein de leurs territoires. Il questionne la cohérence de ces dispositifs, qui émanent parfois de la fusion de plusieurs stratégies locales avec des objectifs de développement agricole et rural qui peuvent être différents.

\section{Dynamique d'émergence des collectifs et nouvelles attentes}

10 L'analyse des différents collectifs agricoles ainsi que l'examen de leurs motivations permettent de mettre en avant trois principaux points qui caractérisent leur recomposition en cours.

11 La premier point soulevé par les études de cas concerne l'émergence de collectifs autour de fonctions ou de missions très précises qui ne relèvent pas uniquement de la sphère productive ou de commercialisation. À l'exception des collectifs d'échange quant aux pratiques visant à réduire les intrants dans le cadre d'une transition agroécologique (Vergote et Tanguy, 2021), les autres études de cas documentent des collectifs travaillant sur la transformation, la logistique, la gestion sanitaire des élevages, la mobilisation des adhérents au sein de leur coopérative, et enfin le développement d'une coopération alimentaire paysanne qui met en œuvre des stratégies d'ancrage territorialisé.

12 Terrieux et al. (2021) analysent les Ateliers de transformation collective qui se sont formés dans la région Occitanie dans l'objectif de capter une valeur ajoutée 
supplémentaire par le recours à la transformation, puis à la commercialisation en circuits courts. Ces ateliers représentent un moyen de diversification agricole permettant de maintenir et de développer les exploitations dans les territoires. Raimbert et Raton (2021) analysent les collectifs logistiques dans les circuits courts alimentaires de proximité de la région Hauts de France. Les auteurs étudient ces collectifs du point de vue de leurs apports logistiques (comment accroître la disponibilité des produits locaux en améliorant la logistique d'approvisionnement ?), sans se limiter au volet commercial fréquemment abordé dans la littérature scientifique. La spécificité de la coopération logistique relève notamment de sa difficulté à être mise en place, car elle implique la mobilisation de matériel favorisant des stratégies individuelles, voire des stratégies de passager clandestin. De plus, la logistique nécessite une diversité d'acteurs avec des objectifs variés alliant une recherche d'efficacité logistique (par la réduction des coûts notamment) et des objectifs territoriaux qui intègrent des logiques non économiques. Gisclard et al. (2021) documentent la naissance de collectifs autour d'un objectif également très spécifique, la gestion sanitaire, grâce à l'étude d'élevages porcins en Corse. Par rapport aux enjeux d'intégration économique de la filière porcine et de sélection génétique, la santé de l'élevage comme justification à la constitution de collectifs est très peu abordée dans la littérature scientifique. L'émergence de ces collectifs de gestion sanitaire répond à un besoin d'une partie des éleveurs corses en matière de visibilité économique (création d'un abattoir permettant la commercialisation de leur viande en Corse) et politique (recherche d'une reconnaissance en tant que partenaire local et interlocuteur officiel vis-à-vis des services de l'État). Vergote et Tanguy, (2021) questionnent les ressorts de constitution et de fonctionnement de collectifs en Bourgogne-Franche-Comté autour de fonctions bien connues à savoir les échanges techniques entre pairs. Il s'agit de collectifs d'échanges spécifiques aux conditions d'adoption des pratiques visant à réduire les intrants dans le cadre d'une transition agroécologique. Amichi et al. (2021) proposent une analyse d'un groupe de jeunes adhérents et salariés dans le Sud-Ouest qui ont été amenés à réfléchir sur les questions de gouvernance de leur coopérative ainsi que sur les facteurs pouvant améliorer leur mobilisation. Les auteurs mettent en évidence un faible environnement de mobilisation au sein de cette coopérative et montrent qu'il est possible de reconstruire un sentiment de mobilisation par le recours aux démarches participatives. Enfin, Noël et al. (2021) questionnent la contribution de deux collectifs dits de coopération alimentaire paysanne, situés en Pays de la Loire (Terroirs 44) et en Wallonie (Paysans-Artisans), à la viabilité des exploitations et des filières au sein des territoires.

13 Le deuxième point caractéristique de la recomposition des collectifs concerne la volonté de ces derniers de se différencier des anciennes formes de la coopération agricole et du modèle agricole dit productiviste. Cette caractéristique a déjà été relevée dans de nombreux travaux et qualifiée de logique d'émancipation vis-à-vis du modèle agricole dominant (Mondy, 2014) ou de volonté de se réclamer de modèles agricoles alternatifs (Maye, 2013 ; Gafsi et Terrieux, 2016).

14 En matière d'organisation de la production et de structuration des filières, plusieurs collectifs se réclament de logiques de diversification et de la vente en circuit court, les amenant à se différencier du modèle agricole qui prône la spécialisation et l'intégration à la filière longue (Terrieux et al., 2021). Nous retrouvons aussi des affirmations fortes au sein de ces collectifs visant à promouvoir des exploitations à taille humaine (Noël et al., 2021), intégrant des pratiques écologiques (Vergote et Tanguy, 2021) et ancrées 
localement permettant des interactions entre les acteurs du territoire (Raimbert et Raton, 2021) et l'expression d'une diversité de formes de solidarités formelles ou informelles (Gisclard et al., 2021). Un mouvement inverse à une agriculture industrielle est ainsi promu par ces collectifs dans l'optique d'évoluer durablement vers une alimentation saine et de qualité, accessible aux consommateurs et permettant de mieux rémunérer les producteurs (Noël et al., 2021).

Le troisième point concerne la convivialité et la socialisation comme motivations qui justifient la création ou la participation aux collectifs. Il est certain que les gains d'efficacité technique et/ou économique restent centraux et constituent la base de ces collectifs, mais les attentes de leurs membres vont largement au-delà pour inclure une forte dimension sociale (liens sociaux, convivialité, appartenance à une histoire, etc.). L'importance de ces liens sociaux a été mise en avant dans des travaux récents comme une composante essentielle de l'engagement dans un mouvement social (Pleyers, 2010 ; Arnsperger, 2016). Les contributions de ce dossier confirment l'importance de ces liens sociaux que ce soit en tant que base de formation d'un collectif, en tant que ciment qui consolide les rapports entre les membres d'un collectif ou comme un besoin de sociabilisation et de convivialité, exprimé en tant que tel par les membres pour rompre avec le caractère solitaire de leurs métiers. Dans ce sens, Gisclard et al. (2021) montrent à quel point les relations sociales de proximité peuvent constituer une échelle pertinente pour structurer efficacement l'action collective en Corse. Vergote et Tanguy (2021), montrent la difficulté de constituer un collectif sans liens sociaux préexistants ou en l'absence de disposition de sociabilisation de la part des membres. Enfin, Amichi et al. (2021) insistent sur l'importance des relations de confiance qui constituent une composante essentielle de la pérennité des collectifs.

\section{Gouvernance et enjeux de pérennité des collectifs}

L'analyse de la gouvernance de différents collectifs au sein de ce dossier thématique permet de révéler un certain nombre de dysfonctionnements pouvant menacer la pérennité des initiatives. Un décalage entre les règles de gouvernance établies et les pratiques réellement observées est constaté au sein des collectifs conduisant à des trajectoires de développement différentes.

Trois types de facteurs expliquant la faible pérennité de certains collectifs peuvent être distingués. Il s'agit premièrement de facteurs d'ordre organisationnel, liés à la faible implication des membres dans le collectif ou au non-respect des règles communes. Terrieux et al. (2021) illustrent par exemple un manque de participation aux postes à responsabilité de nature administrative dans certains collectifs et le non-respect des règles dans d'autres (fraude dans l'utilisation de l'atelier, non-paiement des factures dues à l'atelier). Ces problèmes organisationnels peuvent amoindrir l'efficacité technique des collectifs (défaillance logistique ou manque d'entretien d'un outil de transformation, par exemple) avec une répercussion sur les résultats économiques aussi bien collectifs qu'individuels. Deuxièmement, il s'agit de facteurs de proximité géographique relatifs aux limites spatiales au-delà desquelles il est difficile de constituer un collectif et de maintenir sa dynamique. Ces limites spatiales peuvent concerner également les périmètres d'action des collectifs qui ne coïncident pas toujours avec les limites administratives de certains territoires compliquant leurs rapports avec les institutions, notamment dans le cadre de dispositifs 
d'accompagnement ou de soutien. Gisclard et al. (2021) montrent dans le cas des éleveurs corses le poids déterminant de la proximité géographique dans la formation et la pérennité des collectifs, dans la mesure où elle permet des interactions entre les membres de manière régulière, voire quotidienne. Les mêmes auteurs indiquent que malgré la pertinence de cette échelle spatiale pour les collectifs se pose la question de son articulation avec les logiques de l'action publique sanitaire. Troisièmement, nous retrouvons les facteurs d'ordre social, plus nombreux, qui concernent: i) l'absence d'une histoire commune ou de liens sociaux antérieurs à la création des collectifs, même si cela a été relativisé dans d'autres cas en les considérant moins centraux, ii) la perte de vue des objectifs et des valeurs fondatrices du collectif, généralement associés au développement d'une agriculture alternative, iii) la faible capacité de socialisation des membres et leur inaptitude à faire du collectif. Ces difficultés peuvent entacher fortement le sentiment de confiance et les valeurs de solidarité au sein des collectifs. Vergote et Tanguy (2021) montrent qu'en l'absence d'un sentiment de confiance, les collectifs peuvent être amenés à disparaître malgré la réunion d'un certain nombre de facteurs considérés comme favorables à l'action collective, en particulier: la confrontation des agriculteurs à un même problème agronomique nécessitant un apprentissage collectif, et la présence d'un institut technique pour appuyer et animer le collectif.

18 Ces différentes formes de fragilité et la multiplicité d'acteurs qu'impliquent les collectifs amènent deux articles de ce dossier à réfléchir à la manière de les accompagner afin de renforcer leur pérennité. L'accompagnement des collectifs par un organisme tiers dans le cadre d'une démarche participative constitue une piste privilégiée à condition que le processus participatif soit bien construit et préparé autour de certains prérequis. Vergote et Tanguy (2021) insistent sur l'importance de la confiance dans ce type de démarche qui doit prévaloir à la fois entre les membres du collectif, mais aussi entre le collectif et les animateurs des ateliers participatifs. L'importance de la confiance a également été relevée dans l'article d'Amichi et al. (2021), intégrée dans un concept plus large de "sentiment d'agir» ou «d'empowerment psychologique ». Les auteurs proposent une opérationnalisation du cadre analytique de l'empowerment psychologique sous forme d'un processus participatif. Ils montrent ainsi qu'il est possible de reconstruire un sentiment de mobilisation d'un petit groupe au fil des ateliers participatifs. En cela, ils posent un premier jalon en matière de démarche d'accompagnement qui offre aux différentes formes de collectifs des pistes et des leviers d'action concrets permettant d'améliorer leur gouvernance et leur pérennité.

\section{L'ancrage territorial des collectifs : proximité renouvelée et nouvelles voies de pérennité ?}

L'analyse des dynamiques de repositionnement des systèmes de production - et des démarches collectives qui les portent - s'appuie très souvent sur le concept de transition agroécologique, envisagé notamment au travers d'un usage maîtrisé des ressources, d'une limitation des externalités négatives et de la mise en œuvre de services écosystémiques (Coulon et Meynard, 2011). Au-delà du changement de pratiques, la quête des collectifs agricoles pour une pérennité accrue interroge également leur gouvernance et leur rapport à l'espace. La question de la 
reterritorialisation de l'agriculture (Rieutort, 2009) occupe ainsi une importance croissante dans la littérature scientifique, en particulier dans le champ ces circuits courts de proximité (Praly et al., 2014).

Même si ce point mérite d'être discuté (Plumecocq et al., 2018), le caractère alternatif des collectifs agricoles émergents vis-à-vis du modèle dominant fondé sur des filières industrialisées et déconnectées de leur contexte géographique semble confirmé. Ce caractère est perceptible dans les rapprochements que l'on peut observer entre les producteurs et les consommateurs (Lamine et Chiffoleau, 2012), mais aussi entre les acteurs productifs dans leur ensemble et les autres acteurs du territoire. Les processus de qualification des produits alimentaires issus de ces systèmes productifs peuvent également être envisagés comme alternatifs, dans la mesure où ceux-ci convoquent de nouvelles dimensions, comme l'ancrage local, le lien social et la confiance interpersonnelle (Goodman, 2003), en réponse aux nouvelles attentes des consommateurs et des citoyens.

21 Le dossier thématique constitue une opportunité pour les auteurs d'analyser comment les rapports des collectifs - quelles que soient leurs configurations - au territoire contribuent à leur pérennité et à leur compétitivité, et plus largement à leur inscription dans une dynamique de développement agricole et rural (Marsden et al., 2000). Deux articles insistent plus particulièrement sur cet aspect.

Noël et al. (2021) abordent ce rapport à l'espace à l'aune de deux organisations collectives territorialisées: une structure d'animation en Loire-Atlantique et une structure commerciale en Belgique, qui ont la particularité de s'inscrire dans un positionnement de médiation entre production et commercialisation. Dans cette réflexion, le concept de viabilité est préféré à celui de durabilité, en particulier pour affirmer le caractère alternatif et paysan des démarches collectives analysées et s'émanciper encore davantage d'une logique uniquement productive. L'analyse de la contribution de ces collectifs à la viabilité de l'agriculture est ainsi abordée au prisme de quatre dimensions : le bien-être et la qualité de vie, l'économie, le développement local et l'environnement. Au-delà du caractère multidimensionnel de ces apports, l'article insiste sur le rôle majeur joué par la reterritorialisation des acteurs et des produits.

23 Frayssignes et al. (2021) étudient quant à eux l'évolution de l'agriculture contemporaine et le foisonnement des circuits courts collectifs qui la caractérise. Pour autant, plus que les structures elles-mêmes, c'est leur rapport à l'espace qui est ici au cœur de la réflexion, à travers la propension de ces démarches à contribuer à la relocalisation de l'agriculture. De manière quasi systématique, les chercheurs comme les décideurs tendent à considérer que les circuits courts induisent nécessairement un processus de relocalisation sans jamais réellement définir le terme. À partir d'une revue de la littérature et d'études de cas, les auteurs plaident dès lors pour un recentrage du concept sur son socle spatial et proposent une approche fondée sur deux volets : une relocalisation « objective ", mesurable, privilégiant l'analyse des dynamiques spatiales à l'œuvre; et une relocalisation "vécue", fondée sur les pratiques et les représentations des acteurs.

24 Bien que fondés sur des méthodologies et des approches théoriques différentes, les deux articles se rejoignent dans leurs conclusions sur la nécessité de nuancer les visions trop idéalisées des bénéfices induits par les circuits courts collectifs, en particulier concernant les volets économiques et productifs. Le fait d'interroger la 
dimension territoriale des structures collectives doit en effet permettre de mieux caractériser les autres voies de la pérennité.

\section{Quels dispositifs institutionnels d'accompagnement des collectifs?}

Les modalités d'intervention des acteurs publics dans le secteur agricole et rural ont connu une évolution sensible depuis les années 2000. Cette évolution résulte notamment de la réforme de la PAC de 1999 et la création du second pilier. En parallèle du premier pilier dédié aux aides directes aux agriculteurs, le second pilier est consacré au « développement rural » et repose sur un «menu de mesures » socio-structurelles et agro-environnementales (Lataste et al., 2012) ${ }^{1}$.

Dans ce contexte, les régulations sectorielles laissent de plus en plus de place à des régulations multi-acteurs dans lesquelles les collectivités territoriales se positionnent comme un acteur principal (Berriet-Solliec et al., 2005). Cette évolution a été renforcée dans le cadre de la réforme de la PAC de 2014 qui consacre les Régions comme nouvelles autorités de gestion des fonds européens, leur permettant ainsi d'établir des programmes d'action et des stratégies de développement agricole et rural en fonction des spécificités de leurs territoires (Capt et al., 2014). Dans ce contexte de décentralisation, les collectivités territoriales tentent d'adapter leurs dispositifs d'intervention afin de répondre au mieux aux nouveaux enjeux socio-économiques et environnementaux de l'agriculture.

Les contributions scientifiques de ce numéro thématique documentent la diversité des dispositifs régionaux mis en place afin de s'adapter et d'accompagner en particulier la recomposition des collectifs au sein de leurs territoires. Ces contributions questionnent également la cohérence de ces dispositifs, qui émanent parfois de la fusion de deux stratégies différentes des anciennes régions, avec les objectifs de développement agricole et rural qu'elles se fixent, dans lesquels les structures et initiatives collectives prennent une place importante.

Vollet et al. (2021) présentent une première analyse institutionnelle du rôle joué par les Organismes nationaux à vocation agricole et rurale (Onvar) dans le développement rural en France. Cette catégorie regroupe une diversité d'organismes de par leur histoire, leurs pratiques et leurs modalités d'action dont la reconnaissance s'inscrit dans les modalités de mise en œuvre du Programme national de développement agricole et rural : le PNDAR. Cette reconnaissance leur permet non seulement d'accéder à des financements, mais aussi de participer plus directement à la construction des politiques rurales. L'article étudie les effets de cette institutionnalisation sur leur contribution au développement rural en analysant les mécanismes par lesquels ces organisations collectives répondent à certains objectifs des politiques agricoles, en particulier du PNDAR. Les auteurs montrent que les Onvar apportent une valeur ajoutée en matière de coordination intersectorielle et de partenariats dans le cadre des projets territorialisés qu'ils initient. Cependant, cette coordination est moins présente quand il s'agit de partenariat avec des organismes majoritaires du développement agricole et rural tels que les Chambres consulaires et les Instituts techniques.

Le paysage des politiques publiques en lien avec l'agriculture et l'alimentation a également été marqué par la loi d'Avenir pour l'agriculture, l'alimentation et la forêt de 
2014 et son programme national pour l'alimentation (PNA), qui soutient tout particulièrement l'émergence et la mise en œuvre des projets alimentaires territoriaux (РАT). Ce nouvel instrument marque un tournant dans l'affirmation de la reterritorialisation de l'agriculture, privilégiant plus explicitement le caractère local des initiatives collectives en matière d'alimentation et d'agriculture. Les PAT permettent de rapprocher les producteurs, les transformateurs, les distributeurs, les collectivités territoriales et les consommateurs pour développer l'agriculture locale et promouvoir une alimentation de qualité (Maréchal et al., 2018). Plus récemment encore, et dans une même logique, les États généraux de l'alimentation ont conduit à la mise en place de la loi pour l'Équilibre des relations commerciales dans le secteur agricole et alimentaire et une alimentation saine, durable et accessible à tous (EGAlim).

Dans ce sens, Wallet et Bouroullec (2021) évoquent la façon dont cette évolution du contexte institutionnel favorise le développement de Circuits alimentaires courts de proximité (CACP). Les auteurs analysent comment les régions soutiennent le développement des CACP en prenant pour exemple la région Occitanie. En rappelant l'enchâssement des politiques publiques du niveau européen au niveau régional, puis en analysant plus précisément le rôle joué par les Plans de développement régionaux (PDR) Midi-Pyrénées et Languedoc-Roussillon de la région Occitanie, ils tentent de dégager les avantages et les limites de cette logique régionale des choix d'orientation. Ils montrent ainsi un soutien timide mais plus marqué en Languedoc-Roussillon, mais soulignent que les contours de la future PAC détermineront l'autonomie laissée aux régions pour définir les mesures de soutien aux CACP adaptées aux exigences locales.

31 Au final, les articles de ce dossier montrent le rôle important joué par les collectifs dans le maintien, voire le développement des exploitations et des filières sur les territoires. Bien que la légitimité de ces collectifs dans leurs formes traditionnelles soit fréquemment questionnée (Hervieu et Purseigle, 2011), ils participent malgré tout au renforcement de la capacité des acteurs en matière d'adaptation et d'intégration de différentes formes de changement permettant de mieux amorcer la transition agricole. Les articles portent également des interrogations quant au devenir de ces collectifs et leur capacité à se maintenir dans le temps et dans les territoires. Des clés de compréhension sont identifiées afin de saisir les conditions favorables à la pérennité des collectifs. Des pistes de réflexion sont aussi proposées, permettant de mieux les accompagner. Enfin, ce dossier invite à compléter et à approfondir les travaux sur les collectifs agricoles et les facteurs de leur pérennisation. Il peut s'agir aussi d'investiguer d'autres points insuffisamment étudiés, comme l'apport des collectifs agricoles en matière environnementale (rôles dans l'adoption de pratiques agroécologiques ou dans la réduction de l'empreinte carbone de l'activité agricole, etc.) dont la plus-value nécessite une meilleure caractérisation et quantification. Aussi, l'analyse de la dimension politique des collectifs pourrait être approfondie, en particulier en ce qui concerne leur implication au sein des sphères décisionnelles aux échelles locale comme nationale. 


\section{BIBLIOGRAPHIE}

Allaire G., Assens P., 2002, « Coopération et territoire. Le cas des Coopératives d'utilisation de matériel agricole (Cuma) », Colloque «SYAL: Produits, entreprises, dynamiques locales », 13-14 octobre, Montpellier.

Amichi H., Henninger M.-C., Peltier C., 2021, « Comment mobiliser les jeunes adhérents dans la gouvernance des coopératives agricoles? Mise en pratique d'une démarche d'accompagnement au sein d'une coopérative du Sud-Ouest français ", Développement durable \& territoires, vol. $12, \mathrm{n}^{\circ} 1$, https://journals.openedition.org/developpementdurable/18548.

Arnsperger C., 2016, «Critique existentielle de la croissance économique. Éléments pour une "transition anthropologique" ", Revue interdisciplinaire d'études juridiques, vol. 77, n², p. 73-97, https://doi.org/10.3917/riej.077.0073.

Barraud-Didier V., Henninger M.-C., Anzalone G., 2012, « La distanciation de la relation adhérentcoopérative en France ", Études rurales, n 190, p. 119-130.

Berriet-Solliec M., Déprés C., Trouvé A., 2005, « La territorialisation de la politique agricole en France. Vers un renouvellement de l'intervention publique en agriculture ?» Working Paper, Centre d'économie et sociologie appliquées à l'agriculture et aux espaces ruraux, $14 \mathrm{p}$.

Bouroullec M. D. M., 2020, « Les différentes gouvernances des circuits courts alimentaires de vente en ligne collective ", Économie rurale, vol. 371, n 1, p. 59-75, https://doi.org/10.4000/ economierurale.7521.

Capt D., Lepicier D., Leseigneur A., 2014, « Le rôle des territoires de projets infra-régionaux sur l'agriculture et l'alimentation. Le cas du développement de circuits de proximité ", Géocarrefour, vol. 89, n 89/1-2, p. 105-113, https://doi.org/10.4000/geocarrefour.9436.

Chiffoleau Y., Prevost B., 2012, « Les circuits courts, des innovations sociales pour une alimentation durable dans les territoires ", Norois. Environnement, aménagement, société, n²24, p. 7-20, https://doi.org/10.4000/norois.4245.

Chiffoleau Y., Paturel D., 2016, « Les circuits courts alimentaires “pour tous", outils d'analyse de l'innovation sociale », Innovations, n 2, p. 191-210, https://doi.org/10.3917/inno.050.0191.

Coulon J.-B., Meynard J.-M., 2011, « Vers une agriculture à hautes performances environnementales : état des lieux des voies d'amélioration technique proposées par l'Inra », Innovations agronomiques, $\mathrm{n}^{\circ} 12$, p. 1-15.

Demeulenaere É., Goulet F., 2012, « Du singulier au collectif : agriculteurs et objets de la nature dans les réseaux d'agricultures "alternatives" ", Terrains \& travaux, vol. 1, n 20, p. 121-138, https://doi.org/10.3917/tt.020.0121.

Filippi M., Frey O., Mauget R., 2008, « Les coopératives agricoles face à l'internationalisation et à la mondialisation des marchés ", Revue internationale de l'économie sociale : Recma, n 310, p. 31-51, https://doi.org/10.7202/1021102ar.

Flauraud V., 2005, « La Jeunesse agricole catholique (JAC) », Rives méditerranéennes, n², p. 25-40.

Frayssignes J., Pouzenc M., Olivier-Salvagnac V., 2021, « La relocalisation de l'agriculture : entre dimension spatiale et représentations des acteurs. Le cas des circuits courts collectifs ", Développement durable \& territoires, vol. 12, n 1, https://journals.openedition.org/ developpementdurable/18584. 
Gafsi M., Delevers L., 2015, «Impact des ateliers collectifs de transformation sur les performances socio-économiques des exploitations agricoles », in Mundler P., Valorge F., (éds.) Ateliers de transformation collectifs Transformer collectivement ses produits agricoles dans les territoires, Dijon, Educagri Éditions,p. 235-258.

Gafsi, M., Terrieux A., 2016, « Ateliers de transformation collectifs. Le rôle des proximités dans les stratégies des agriculteurs ", in Mundler P. (dir), Alimentation et proximités. Jeux d'acteurs et territoires, Dijon, Educagri Éditions, p. 249-265.

Gerbaux F., Muller P., 1984, «La naissance du développement agricole en France », Économie rurale - Agricultures Alimentations Territoires, $\mathrm{n}^{\circ}$ 159, p. 17-22, https://www.persee.fr/doc/ ecoru_0013-0559_1984_num_159_1_3019.

Gisclard M., Devleeshouwer P., Charrier F., Casabianca F., 2021, « Action collective et gestion sanitaire des élevages porcins en Corse », Développement durable \& territoires, vol. 12, n 1, https:// journals.openedition.org/developpementdurable/18618.

Goodman D., 2003, « The quality "turn" and alternative food practices: reflections and agenda », Journal of Rural Studies, $\mathrm{n}^{\circ} 19, \mathrm{p} .1-7$.

Hervieu B., 2002, « La multifonctionnalité de l'agriculture : genèse et fondements d'une nouvelle approche conceptuelle de l'activité agricole », Cahiers Agricultures, vol. 11, nº 6, p. 415-419.

Hervieu B., Purseigle F., 2011, « Des agricultures avec des agriculteurs, une nécessité pour l'Europe », Revue Projet, n 2, p. 60-69, https://doi.org/10.3917/pro.321.0060.

Lamine C., Chiffoleau Y., 2012, « Reconnecter agriculture et alimentation dans les territoires : dynamiques et défis », Pour , n 215-216, p. 85-92, https://doi.org/10.3917/reru.123.0327.

Lataste F., Berriet-Solliec M., Trouvé A., Lépicier D., 2012, « Le second pilier de la Politique agricole commune : une politique à la carte ", Revue d'économie régionale \& urbaine, $\mathrm{n}^{\circ} 3$, p. 327-351, https://doi.org/10.3917/reru.123.0327.

Lucas V., Gasselin P., Thomas F., Vaquié P.-F., 2014, « Coopération agricole de production : quand l'activité agricole se distribue entre exploitation et action collective de proximité ", in Gasselin P., Choisis J.-P., Petit S., Purseigle F., Zasser S., (éd,), L'agriculture en famille : travailler, réinventer, transmettre, Les Ulis, EDP Sciences, p. 201-222.

Maréchal G., Spanu A., 2010, « Les circuits courts favorisent-ils l'adoption de pratiques agricoles plus respectueuses de l'environnement? ", Courrier de l'environnement de l'Inra, n 59, p. 33-45.

Maréchal G., Noël J., Wallet F., 2018, « Les projets alimentaires territoriaux (PAT) : entre rupture, transition et immobilisme? ", Pour, n 2, p. 261-270.

Marsden T., Banks J., Bristow G., 2000, « Food supply chain approaches, exploring their role in rural development », Sociologia Ruralis, vol. 40, $\mathrm{n}^{\circ}$ 4, p. 424-438.

Maye D., 2013, « Moving Alternative Food Networks beyond the Niche », International Journal of Sociology of Agriculture and Food, vol. 20, n 3, p. 383-389.

Mondy B., 2015, « Des premiers Cuma de diversification aux ATC, vers une évolution des formes coopératives de production ", in Mundler P., Valorge F. (dir), Ateliers de transformation collectifs. Enjeux et outils pour réussir, Dijon, Educagri Éditions, p. 23-37.

Mundler P., Valorge F., 2015, Ateliers de transformation collectifs. Enjeux et outils pour réussir, Dijon, Educagri Éditions.

Noël J., Margetic C., Lanzi F., Dogot T., Maréchal K., 2021, « De l'importance des structures collectives territorialisées pour consolider la viabilité des circuits alimentaires de proximité. Le 
cas de Terroirs 44 et de Paysans-Artisans ", Développement durable \& territoires, vol. $12, \mathrm{n}^{\circ} 1$, https://journals.openedition.org/developpementdurable/18679.

Pleyers G., 2010, Alter-globalization: Becoming actors in a global age, Cambridge, Polity.

Plumecocq G., Debril T., Duru M., Magrini M., Sarthou J., Therond O., 2018, « Caractérisation socio-économique des formes d'agriculture durable ", Économie rurale, n 363, p. 99-120, https:// doi.org/10.4000/economierurale.5430.

Praly C., Chazoule C., Delfosse C., Mundler P., 2014, « Les circuits de proximité, cadre d'analyse de la relocalisation des circuits alimentaires ", Géographie, Économie, Société, vol. 16, p. 455-478, https://doi.org/10.3166/ges.16.455-478.

Raimbert C., Raton G., 2021, « Collectifs logistiques et territoires dans les circuits courts alimentaires de proximité : la robustesse de la coopération analysée au prisme des communs », Développement durable \& territoires, vol. 12, n 1, https://journals.openedition.org/ developpementdurable/18754.

Richard F., Chevallier M., Dellier J., Lagarde V., 2014, « Circuits courts agroalimentaires de proximité en Limousin : performance économique et processus de gentrification rurale ", Norois. Environnement, aménagement, société, n² 230, p. 21-39, https://doi.org/10.4000/norois.4997.

Rieutort L., 2009, « Dynamiques rurales françaises et reterritorialisation de l'agriculture », L'Information géographique, vol. 73, p. 30-48.

Terrieux A., Gafsi M., Fiaschi U., 2021, « Dynamiques de création et trajectoires d'Ateliers de Transformation Collectifs (ATC) dans la région Occitanie », Développement durable \& territoires, vol. 12, n 1, https://journals.openedition.org/developpementdurable/18804.

Van Dam D., Lagneaux S., Nizet J., Streith M., 2017, Collectifs en agriculture bio (Les) : Entre idéalisation et réalisation, Dijon, Educagri Éditions.

Van Der Ploeg J.-D., 2014. Les paysans du XXI ${ }^{e}$ siècle. Mouvements de repaysanisation dans l'Europe d'aujourd'hui. Paris, Éditions Charles Léopold Mayer, 214 p.

Van Der Ploeg J.-D., Laurent C., Blondeau F., Bonnafous P., 2009, « Farm diversity, classification schemes and multifunctionality ", Journal of environmental management, vol. 90, p. S124-S131.

Vergote M.-H., Tanguy C., 2021, « Collectifs d'échanges de pratiques pour écologiser l'agriculture : éclairer les difficultés d'une approche volontaire », Développement durable \& territoires, vol. 12, $\mathrm{n}^{\circ}$ 1, https://journals.openedition.org/developpementdurable/18861.

Vincq J.-L., Mondy B., Fontorbes J.-P., 2010, « La construction de la qualité fiable dans les réseaux alimentaires de proximité », Économie rurale, n 318-319, p. 5-19.

Vollet D., Bossuat H., Gasselin P., Labarthe P., Wallet F., Barbarot A-S., Filippi M., Rousselière D., 2021, « La valeur ajoutée des « Organismes nationaux à vocation agricole et rurale » (Onvar) au développement territorial », Développement durable \& territoires, vol. 12, $\mathrm{n}^{\circ} 1$, https:// journals.openedition.org/developpementdurable/18921.

Wallet F., Dantas Machado Bouroullec M., 2021, « Politique publique et développement des chaînes alimentaires courtes de proximité : une analyse des PDR de la région Occitanie ", Développement durable \& territoires, vol. 12, n 1, https://journals.openedition.org/ developpementdurable/18989. 


\section{NOTES}

1. Détail des mesures: Nègre F., 2021, "Second pilier de la PAC: la politique de développement rural », Fiches techniques sur l'Union européenne: https:// www.europarl.europa.eu/factsheets/fr/sheet/110/second-pilier-de-la-pac-la-politiquede-developpement-rural.

\section{AUTEURS}

\section{HICHEM AMICHI}

Hichem Amichi est enseignant-chercheur en agroéconomie. Il travaille sur les questions de structuration organisationnelle des filières agricoles et agroalimentaires en France et dans les pays du Sud. Il analyse en particulier le lien entre les rapports contractuels (formels et informels) développés par les acteurs au sein des filières et leur capacité à intégrer différentes formes d'innovation, dans le cadre notamment de la transition agroécologique et solidaire. LISST-Dynamiques rurales, université de Toulouse, UMR 5193, CNRS UT2J, EHESS, ENSFEA, INP PURPAN, Toulouse, France hichem.amichi@purpan.fr nºrcid : 0000-0003-0963-7086

\section{JULIEN FRAYSSIGNES}

Julien Frayssignes est enseignant-chercheur en géographie. Il travaille sur le développement des territoires ruraux et sur les dynamiques d'ancrage et de relocalisation des activités agricoles et agroalimentaires, en particulier au travers des démarches de qualification portées par les filières (signes officiels, démarches privées). Il est à l'origine de la création d'une chaire d'enseignement et de recherche dédiée à cette question (In'FAAQT : Innover dans les filières agricoles, agroalimentaires, la qualité et les territoires) LISST-Dynamiques rurales, université de Toulouse, UMR 5193, CNRS UT2J, EHESS, ENSFEA, INP PURPAN, Toulouse, France

https://infaaqt.com/

julien.frayssignes@purpan.fr

\section{MARIE-CHRISTINE HENNINGER}

Marie-Christine Henninger est maître de conférences en sciences de gestion, elle étudie notamment les coopératives agricoles à travers les relations qu'elles entretiennent avec leurs adhérents et les enjeux autour de leur fidélité ou de leur participation à la gouvernance de ces structures.

LISST-Dynamiques rurales, université de Toulouse, UMR 5193, CNRS UT2J, EHESS, ENSFEA, INP PURPAN, Toulouse, France

marie-christine.henninger@univ-tlse2.fr

nºrcid : 0000-0003-1850-1324 


\section{ARNAUD BUCHS}

Arnaud Buchs est maître de conférences en économie. Ses recherches articulent une approche en économie écologique et institutionnaliste et le recours au terrain. Elles portent sur l'analyse des politiques et des règles qui encadrent la régulation des ressources en eau et leurs usages. Il est corédacteur en chef de la revue Développement durable \& territoires.

Sciences Po Grenoble, laboratoire GAEL (UMR CNRS, Inrae, Grenoble INP, UGA)

arnaud.buchs@sciencespo-grenoble.fr

n Orcid : 0000-0003-1850-1324 